\title{
Nuevas estrategias de valorización turística en el litoral norte del Partido de General Pueyrredón (Provincia de Buenos Aires, Argentina)
}

\author{
MARÍA CECILIA RIGONAT, PATRICIA ALEJANDRA MORRELL
}

Grupo de Investigación Ambientes Costeros, Departamento de Geografía, Facultad de Humanidades, Universidad Nacional de Mar del Plata, Argentina

crigonat@gmail.com; pmorrell@mdp.edu.ar

\section{RESUMEN}

El litoral marplatense reconoce al turismo de sol y playa como el principal factor de valorización del territorio, sin embargo, este proceso abrigó fuertes desigualdades. El sector norte del partido de General Pueyrredón ha cumplido el rol de absorber las externalidades derivadas de la construcción del puerto y del vertido de los efluentes de la ciudad. El objetivo de este trabajo apunta a interpretar los procesos de valorización diferencial del territorio, que han dado lugar a funcionalizaciones territoriales específicas, en el marco de la actividad turística. La metodología de trabajo apunta a identificar las diferentes actuaciones espaciales $-\mathrm{y}$ las intencionalidades de quienes las llevaron a cabo- desde una mirada diacrónica-sincrónica. Si bien a lo largo de siglo XX se asistió a un paulatino proceso de desvalorización y deterioro ambiental del litoral norte algunas intervenciones del sector público y privado plantean el interrogante sobre una posible refuncionalización turística.

PALABRAS ClaVE: espacio litoral; turismo; Mar del Plata.

Recepción: 20/02/2018 | Evaluación: 09/09/2018 | Aceptación: 07/10/2018 


\section{ABSTRACT}

NEW STRATEGIES FOR TOURISM VALUATION IN THE NORTH LITORAL OF THE GENERAL PUEYRREDÓN DISTRICT (PROVINCE OF BUENOS AIRES, ARGENTINA)

The coast of Mar del Plata recognizes sun and beach tourism as the main factor of valorization of the territory, however, this process harbored strong inequalities. The northern sector of the General Pueyrredón district has fulfilled the role of absorbing externalities derived from the construction of the port and the discharge of effluents from the city. The aims of this study is to interpret the processes of differential valuation of the territory, which have given rise to specific territorial functionalizations, within the framework of tourism activity. The methodological approach aims to identify the different spatial actions - and the intentions of those who carried them out from a diachronic-synchronous perspective. Although, during the twentieth century, a gradual process of devaluation and environmental deterioration of the north coast was attended, some public and private sector interventions raise the question of a possible tourist refunctionalization.

KEYwORDS: coastal zone; tourism; Mar del Plata. 


\section{RESUMO}

NOVAS ESTRATÉGIAS DE AVALIAÇÃO DO TURISMO NO LITORAL NORTE DO PARTIDO GERAL PUEYRREDÓN (PROVÍNCIA DE BUENOS AIRES, ARGENTINA)

A costa de Mar del Plata reconhece o turismo de sol e praia como o principal fator de valorizaçáo do território, no entanto, esse processo abrigava fortes desigualdades. O setor norte do partido General Pueyrredon cumpriu o papel de absorver externalidades derivadas da construçáo do porto e da descarga de efluentes da cidade. O objetivo deste trabalho tem como objetivo interpretar os processos de valoração diferencial do território, que deram origem a funcionalizaçôes territoriais específicas, no âmbito da atividade turística. A metodologia de trabalho tem como objetivo identificar as diferentes açóes espaciais - e as intençóes daqueles que as realizaram - sob uma perspectiva diacrônico-síncrona. Embora durante o século XX tenha ocorrido um processo gradual de desvalorização e deterioração ambiental do litoral norte, algumas intervençóes dos setores público e privado levantam a questão de uma possível re-funcionalização turística.

PALAVRAS-CHAVE: espaco costeiro; turismo; Mar del Plata. 


\section{Introducción}

Los espacios litorales constituyen áreas de características peculiares dada su condición de interfase entre la atmósfera, el continente y el mar. La potencialidad en cuanto a los recursos que abrigan los ha convertido en el escenario de múltiples presiones y conflictos.

Este trabajo toma como recorte territorial la porción norte del partido de General Pueyrredón (provincia de Buenos Aires-Argentina) con la finalidad dar cuenta de los procesos de funcionalización turística materializados a lo largo del tiempo, que han asignado un rol marginal a este espacio. En los últimos diez ańos esta tendencia parece revertirse, en función del agotamiento y saturación de otros sectores de la ciudad.

\section{Marco teórico metodológico}

Se entiende por litoral a una superficie que involucra tanto a elementos del medio natural marino, continental y atmosférico como al conjunto de artefactos producidos por el hombre y las interacciones resultantes entre los mismos. (Villar, 2000). Se trata de un espacio complejo, de límites imprecisos, de extrema sensibilidad y fragilidad ambiental, dinámico y con diversas potencialidades productivas, recreativas y de instalación humana.

Siguiendo a Sánchez (199I) la multiplicidad de actividades económicas y sociales, que se pueden desarrollar en los espacios litorales, da lugar a conflictos entre actores y agentes económicos. La atribución de un uso determinado será la resultante de un proceso histórico y de relaciones de poder entre los agentes (públicos y privados) que pugnarán por materializar determinadas actuaciones espaciales que conllevan procesos de funcionalización específicos para cada fragmento territorial.

En el caso del turismo, es posible distinguir entre espacios de ocio y espacios complementarios. Los espacios de ocio, son espacios de acceso gratuito y de dominio público, representados por el conjunto de recursos geofísicos involucrados en estas prácticas (playa, mar, paisaje). Los espacios complementarios son aquellos en los cuales se ubican los prestadores de los servicios (gastronomía, tendido de sombra, recreación, alojamiento) y, al mismo tiempo, constituyen el ámbito en cual se efectiviza la apropiación del beneficio, requieren de una particular atención por parte del estado (Municipal y Provincial) para su puesta 
en valor y conservación de los atributos que generaron y generan la demanda turística. (Sánchez, I99I).

En este marco de análisis se han identificado las principales actuaciones espaciales realizadas a lo largo del tiempo en el litoral norte del partido de General Pueyrredón, poniéndolas en el contexto general de los procesos de valorización prevalecientes en el litoral del partido y especialmente en la ciudad cabecera (Mar del Plata). Con esta finalidad se ha recurrido al análisis e interpretación de trabajos realizados con anterioridad (Morrell, 2002, Rigonat, 2012) y de autores que han dado cuenta, desde diversas ópticas, de las intervenciones realizadas en el área litoral, tales como Villar (2000), Pastoriza (20II), Cicalese (200I), Lagrange (1993), Sebreli (1970). También se han consultado pliegos de licitaciones y boletines municipales (Municipalidad de General Pueyrredón).

Esta doble mirada diacrónica/sincrónica nos ha permitido reinterpretar las actuaciones espaciales materializadas en el área en cuestión (litoral norte del partido de General Pueyrredón) que sistemáticamente asignaron el rol de absorber las externalidades de las actividades económicas realizadas en otros puntos de la costa y de la ciudad. De la misma forma, esta metodología nos ha permitido resignificar el actual interés por intervenir en el área, puesto de manifiesto por actores públicos y privados.

\section{El litoral marplatense}

Siguiendo a Siragusa (1984) el frente litoral del partido de General Pueyrredón, desde el punto de vista geomorfológico, puede dividirse en 3 tramos (ver mapa I):

1. Desde el límite norte del partido hasta Punta Iglesia, caracterizado por un acantilado formado por rocas sedimentarias, friables, perfectamente definido, que varía entre los 3 y 15 metros, con playas angostas y con una zona de rompiente muy cerca del barranco, especialmente en situaciones de pleamar.

2. Desde Punta Iglesia hasta Punta Mogotes, la costa está conformada por el extremo oriental del bloque Tandilia, con afloramiento de cuarcitas que forman rompientes junto al mar y una topografía ondulada. Se desarrollan bahías y ensenadas que acumulan arena, como en las playas Popular, Bristol, Chica y Grande. 
3. Desde Punta Mogotes hasta el límite sur del partido de General Pueyrredón, con relieve fuertemente ondulado debido a la presencia de cursos de agua, que fueron modelando sus valles fluviales en un proceso de levantamiento de la pampa interserrana. La acción del mar se hizo efectiva en una etapa reciente, situación que llevó a la formación de un acantilado vertical que alcanza en algunos lugares los 30 metros. En otros el acantilado disminuye, formándose pequeñas ensenadas que dan lugar a playas más amplias.

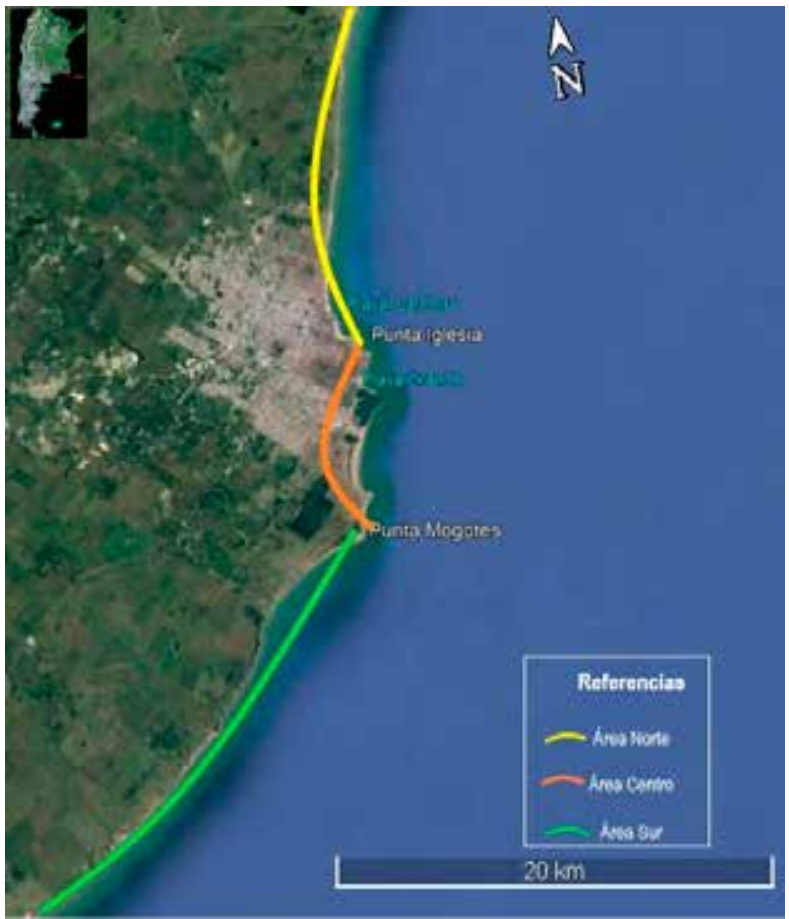

Fuente: elaboración propia.

Mapa 1. Áreas del Litoral de Mar del Plata

La construcción de las escolleras requeridas para la instalación del puerto de Mar del Plata hacia principios del siglo XX, resultó en una interrupción de la corriente de deriva litoral, que tiene una dominancia en la dirección sur-norte, y modificó los procesos de acumulación y erosión de arena en el partido. De esta forma, las playas ubicadas al sur del puerto, especialmente Punta Mogotes, vieron incrementar sus 
dimensiones mientras que en las ubicadas al norte los procesos erosivos se intensificaron. Esta situación dio lugar a la construcción de obras de defensa costera que significaron un desplazamiento de la problemática en el sentido sur-norte afectando incluso a municipios vecinos. (Lagrange, 1993)

La primera funcionalización turística del litoral del Partido de General Pueyrredón aconteció hacia fines del siglo XIX: estaba focalizada en las actuales Playas Bristol y Popular, orientada a satisfacer las necesidades de ocio de las acomodadas clases altas porteńas, que intentaron recrear en este espacio los balnearios europeos. En las primeras décadas del siglo $\mathrm{XX}$, dada la concurrencia de otros estratos sociales más numerosos, los sectores más elitistas se trasladaron hacia Playa Grande (ubicada al sur de las anteriores). La zona de Playa La Perla, ubicada al norte de la Bahía Bristol, en cambio, siempre albergó a una clase menos pudiente que las anteriores y a la población residente.

Posteriormente, hacia mediados del siglo XX, en un contexto de ascenso social a nivel nacional, otros grupos de población, concurrieron al balneario y por ende se intensificó el uso de toda la porción central de la costa marplatense.

En líneas generales, el tramo ubicado entre Punta Iglesia y Punta Mogotes fue el escenario de los continuos procesos de valorización costera como respuesta a la democratización social de Mar del Plata como centro balneario (Pastoriza, 20II). Esta porción de la costa sufrió tempranas modificaciones a su fisonomía natural (ramblas, avenidas costaneras, urbanización, balnearios) acompañadas de obras para la recuperación de playas (escolleras, obras de defensa costera, refulado) con la finalidad de satisfacer los requerimientos de una clientela cada vez más diversificada. Hacia la década de los '70 se observa una especialización en la oferta: sol, playa y diversión que valoriza particularmente el tramo central. La ciudad se convierte en monoproductora, cambiando drásticamente su traza, especialmente con la construcción de edificios en torre, a lo largo de la línea urbana costera, que actúa como barrera entre la ciudad y el mar (y en ciertos sectores céntricos, como Playa Popular, proyectan sombra durante la tarde). En los ' 80 , se asiste al surgimiento de las playas del sur (que vieron incrementar su superficie gracias a las escolleras del puerto), iniciadas con el cuestionado complejo de Punta Mogotes y posteriormente, en los ' 90 , el sur del partido se convierte en 
el escenario de las playas privadas y emprendimientos que apuntan a un turismo de mayor poder adquisitivo. (Rigonat, 20I2).

Recapitulando, a lo largo del tiempo puede verificarse la funcionalización de distintos tramos del litoral marplatense, que si bien tienen en común, la especialización en el turismo de sol y playa, los usuarios que consumen ese espacio de ocio son diferentes y por tanto las inversiones -en calidad y cantidad- son también disímiles. El Estado (Municipal y Provincial) ha desempeñado un rol fundamental en este proceso de diferenciación turística (obras de defensa costera, licitación de balnearios y prestación de servicios, etc.).

\section{El norte, un espacio postergado}

Según Lagrange (1993), a principios del siglo XX, se conocía con el nombre generalizado de La Perla a todo el sector norte de la ciudad (es decir el tramo de costa que va desde Punta Iglesia hasta el arroyo La Tapera, hoy límite del ejido urbano), con el tiempo esta denominación se fue adjudicando a un sector cada vez más acotado.

En la actualidad, el Ente Municipal de Turismo distingue tres sectores que conforman las playas del litoral norte de la ciudad (ver mapa 2): I) La Perla, comprendido entre Punta Iglesia y la calle Necochea; 2) Perla Norte, abarca desde calle Necochea hasta Constitución y 3) Playas del Norte o Playas Camet, desde la Av. Constitución hasta el Arroyo La Tapera.

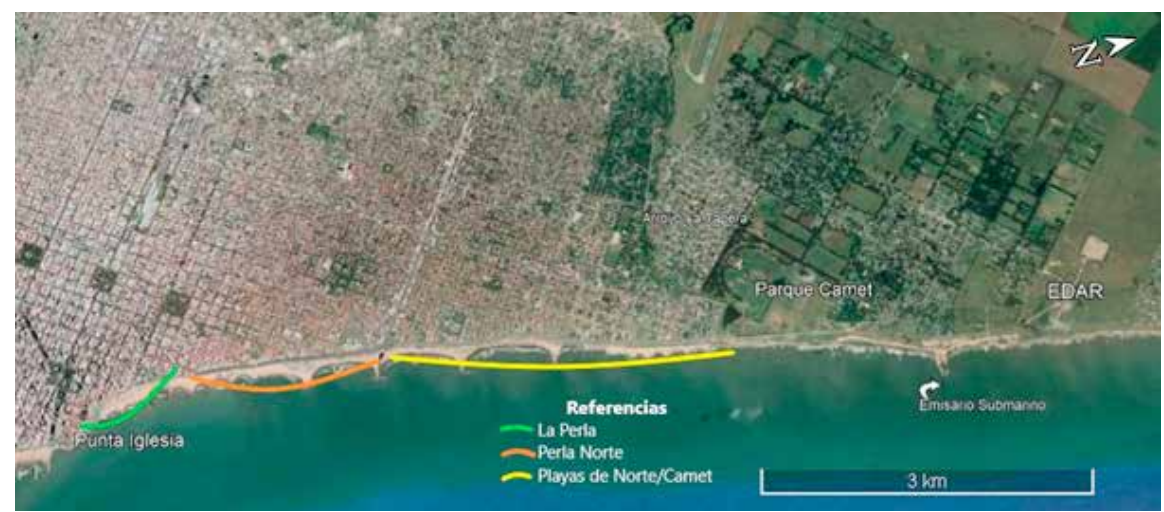

Mapa 2. Sectores del Litoral Norte Fuente: elaboración propia. 
Hacia fines del siglo XIX ya se habían instalado algunos hoteles y balnearios en la zona de la actual Playa La Perla, a los que se accedía a través de escalinatas de madera, que fueron destruidos por sucesivos temporales. Este sector era frecuentado por los trabajadores residentes en la ciudad y por algunos turistas que acudían en búsqueda de tranquilidad. Las obras de defensa costera tardaron en llegar, recién en la década del ' 30 la Dirección de Hidráulica de la provincia de Buenos Aires diseña y ejecuta obras de defensa costera, en el marco de un plan inversión para la costa marplatense (Lagrange;1993).

Hacia principios del siglo XX, el Municipio autorizó la instalación de un punto de descarga al mar de los efluentes cloacales de la ciudad, en un sector ubicado unos 2000 metros al norte del antiguo balneario La Perla. Esta acción muestra claramente la escasa significación que se le daba desde la órbita municipal al sector, considerándolo como sumidero de los desechos cloacales e intensificando aún más el estancamiento del área. (Morrell, 2002).

En el verano de 1937 se inaugura la Colonia de Vacaciones Alfonsina Storni (dependiente del Consejo Nacional de Educación) en un predio cercano al punto de vertido de efluentes. Esto motivó a que el municipio impulsara el traslado del punto de descarga de los efluentes cloacales unos metros más hacia el norte (quedando fuera del ejido urbano).

Sebreli (1970), se refiere a este sector como una zona poco poblada, sin impulso edilicio, considerando a las playas de La Perla como "la playa típica de las familias pequeño burguesas que buscan tranquilidad y no tienen mayores pretensiones de prestigio social, sus concurrentes son frecuentemente propietarios de las casitas de los alrededores, se conocen entre sí desde muchas temporadas y hacen la misma vida de barrio al margen del tumulto del centro, similar a la que llevan en el suburbio de Buenos Aires donde viven" (Sebreli; 1970:87).

La década de los ' 80 marca un quiebre en los mencionados procesos de desvalorización ya que confluyen una serie de acciones emprendidas desde la órbita estatal que marcarán un cambio en esta tendencia.

La intensificación del uso de las playas céntricas llevó a que desde la órbita oficial se pergeñaran nuevos proyectos, valorizando nuevos tramos costeros con la finalidad de descongestionar el área céntrica. En este contexto se inaugura el complejo Punta Mogotes, ubicado al sur del puerto marplatense y, posteriormente, el municipio apunta al sector 
de playas La Perla. Hacia mediados de los '8o se decide la construcción de un complejo de balnearios que abarcaría un perímetro de 800 metros. Para la elaboración del proyecto se convocó a una licitación a nivel nacional (resultando seleccionado el proyecto presentado por el estudio de arquitectura de Testa, Genoud y Álvarez Rojas). Del proyecto original, el único balneario que no se concretó fue el número 5 (hoy Unidad Turística Fiscal), cuya edificación estaba ligada a una defensa costera que quedó inconclusa. (Cicalese, 200I).

En segundo lugar, también desde el gobierno municipal se toma conciencia acerca de la necesidad de contar con algún tipo de tratamiento de las aguas residuales marplatenses. Es así que en 1987 se inician las obras de construcción de una planta de tratamiento primario y separación de material flotante y desinfección parcial, inaugurándose en 1989. (Morrell, 2002). Una década más tarde cobra fuerza la idea de anexar un emisario submarino, que por diversas razones (económicas y técnicas), recién queda materializado en el año 20I4. Esta obra, sumada a nuevas inversiones en el tratamiento de los efluentes cloacales, induce a una mejora en las condiciones ambientales del litoral norte de la ciudad.

Por último, el Departamento de Obras Marítimas de la provincia de Buenos Aires diseñó y comenzó a ejecutar un proyecto integral de obras de defensa costera que abarcaban el sector comprendido desde La Perla hasta Camet. El mismo consistía en remodelar las obras de defensa existentes y construir un tipo de rompeolas curvo, con la finalidad de favorecer la acumulación de arena (Lagrange, 1993). Si bien estas obras tardaron en completarse por razones presupuestarias (quedan finalizadas recién para la década de los '9o), resultaron efectivas, registrándose en la actualidad un notorio aumento de la superficie de playa.

\section{La situación actual}

\section{Sector Playas La Perla}

Este conjunto de playas es el más cercano al centro de la ciudad y también el de mayor grado de urbanización y oferta de servicios. En la actualidad está conformado por el Balneario No I (Alfonsina), Balneario $\mathrm{No}_{2}$ (Saint Michel), Balneario No 3 (San Sebastián) y el Balneario No 4 (Alicante). Los mismos están concesionados y cuentan con servicios de alquiler de carpas y sombrillas, baños públicos, gastronomía, comercios 
de abastecimiento, vestuarios, wifi, canchas para la práctica de deportes, actividades recreativas y rampas de permiten el acceso a personas con movilidad reducida. Todo este sector está en proceso de remodelación desde el año 20I6, que va desde una renovación del equipamiento de playa hasta la instalación de piscinas.

La Unidad Turística Fiscal (UTF) número 5 es el único espacio de playa que había quedado sin concesionar, para lo cual en el año 20I7, se llama a licitación. Según el pliego de licitación (MGP, 20I7) esta intervención se propone reconvertir el área en una oferta turística anual, priorizando los accesos peatonales (tanto de circulación en el borde de la barranca y paralela a la avenida como también la accesibilidad a la playa) y la provisión de servicios de acceso público y actividades comerciales, recreativas y gastronómicas que permitan la utilización de este espacio aún en época invernal.

El proyecto presentado por el estudio de Arquitectura Mariani-Pérez Maraviglia-Cañadas, para la firma Playa Soleada S.A., fue seleccionado por el Ente Municipal de Turismo como la más conveniente, aunque aún no ha sido adjudicado.

\section{Sector Playas Perla Norte}

Este sector comprende el tramo costero limitado por las calles Necochea y la Avenida Constitución. Se verifica la existencia de balnearios que prestan servicios básicos (de menor diversidad y calidad que los anteriores) que alternan con amplios espacios de playa carentes de servicios.

Los balnearios número I (Perla Norte), número 2 (Bahía Bonita) y número 3 (Puerto Cardiel), fueron licitados hacia fines de la década de los '9o. Dentro de la oferta de servicios se destaca el alquiler de carpas y sombrillas, vestuarios, gastronomía, canchas de vóley y fútbol 5 , estacionamiento y baños públicos.

El balneario Perla Norte, se especializa en la atención a personas con discapacidad para lo cual cuenta con una extensa rampa de acceso, que a través de senderos de madera permite llegar hasta el borde del mar, y equipamiento para personas con escasa movilidad (sillas de ruedas, silla anfibia, sector de sombra con piso de madera) y personal técnico especializado. En el año 20I4 fue distinguido en accesibilidad por el Ministerio de Turismo de la Nación y Secretaría de Turismo de la Provincia de Buenos Aires. 
Los balnearios Puerto Cardiel y Bahía Bonita comparten el espacio de estacionamiento, las rampas de acceso y los baños púbicos (servicio de prestación obligatoria) que se encuentran entre los dos balnearios.

Los espacios denominados Unzué I y 2 (por encontrarse frente al Instituto Saturnino Unzué) no han sido concesionados: el acceso se realiza a través de escaleras de mampostería de unos 30 escalones con un pequeño descanso en el medio o a través de los balnearios vecinos. Sólo cuentan con un pequeño puesto de comidas rápidas y los baños públicos son muy precarios debiendo utilizarse los existentes en los balnearios vecinos. La falta de servicios ha sido puesta en evidencia en continuos reclamos de los usuarios, que llevaron a que en 20I5, interviniera la Defensoría del Pueblo, a través del Programa Defensoría del Turista, solicitando al municipio la instalación de baterías de baños de uso público y gratuito.

Durante la temporada 2017-20I8, el gobierno de la provincia de Buenos en el marco del programa Mar del Plata te hace Feliz, brindó servicios en distintos puntos de la ciudad. Este programa comprendía la prestación de servicios en algunas playas de Mar del Plata y la dotación de un bus turístico que partía del centro de la ciudad y trasladaba a las personas en forma gratuita a distintas playas, promocionadas por la provincia, entre ellas las ubicadas en el litoral norte.

En este contexto la denominada playa del Museo (por estar ubicada frente al museo de Arte Contemporáneo, inaugurado en 20I3), fue dotada de equipamiento turístico. El conjunto de obras realizadas comprendió la remodelación de las escalinatas que comunican el borde de la barranca con la playa, la instalación de unas 500 sombrillas (con capacidad para 4 personas), baños públicos, duchas exteriores y lockers con cargadores de celular, puestos de venta de bebidas, agua caliente para mate y wifi gratuito brindado por el Ministerio de Modernización de la Nación.

Cabe aclarar que recientemente (en mayo de 2018) el Poder Ejecutivo Municipal presentó un proyecto para crear y licitar la unidad turística fiscal (UTF) denominada Playa Museo MAR, con la finalidad de dotar a la zona de servicios básicos para el turista, con plazos de licitación de 5 años (a diferencia de los otorgados con anterioridad que iban de los io a I2 años) y cánones más bajos. 


\section{Sector Playas del Norte o Playas Camet}

Este sector está comprendido entre la avenida Constitución y el arroyo La Tapera, es el tramo más alejado del centro y también, más cercano al punto de vertido de los efluentes cloacales (Emisario Submarino, Estación Depuradora de Aguas Residuales -EDAR-).

El único balneario licitado que ofrece todos los servicios es Costa del Sol (licitado hacia fines de los ‘9o). En este sector las playas son relativamente amplias gracias a la acción de acumulación de arena de los espigones en T. Ofrece alquiler de carpas y sombrillas, vestuarios, duchas, servicio gastronómico y estacionamiento. Además de espacios para algunas actividades recreativas como vóley y juegos infantiles. Se accede a este balneario por una escalera de mampostería y por la bajada del estacionamiento. Los baños públicos se encuentran en el extremo sur del balneario.

La playa Constitución, ubicada entre la avenida homónima y el balneario Costa del Sol, carece de servicios, pero recibió la inversión de la provincia de Buenos Aires. En este caso no se brindó el servicio de sombra (sólo un pequeño espacio destinado a adultos mayores), sino que se la orientó a actividades recreativas y deportivas (incluyendo un escenario para espectáculos) Se incorporó una dotación de baños, un puesto de atención médica, conexión a internet.

Al norte del Balneario Costa del Sol se han constituido algunos balnearios con permisos precarios (de una duración de una o dos temporadas) por parte del municipio que sólo ofrecen servicios de venta de bebidas y comidas rápidas, baños químicos, alquiler de algunas sombrillas, mesas y sillas y espacio para estacionamiento, tales como Sun Rider y El Cielo (que sólo estuvo una temporada). Resulta notoria la disminución de la calidad y cantidad de servicios y equipamiento turístico a medida que se avanza hacia el norte. En la actualidad estas playas son frecuentadas principalmente por vecinos de la zona o residentes marplatenses procedentes de barrios periféricos y algunos turistas, que acuden a estas playas en busca de tranquilidad. (Gasperotti, 20I8).

En este contexto, en el año 2018, desde el Gobierno Municipal se ha propuesto un proyecto que pretende crear nuevas unidades turísticas fiscales (Playa Constitución, Beltrán Norte y Beltrán Sur), con plazos de licitación de cinco ańos y reducción de cánones con la finalidad de proveer de servicios a todo este tramo de costa. 


\section{A modo de conclusión}

El litoral norte de la ciudad de Mar del Plata constituye un área de características particulares ya que está constituida por un acantilado fácilmente erosionable que dificulta el acceso a unas estrechas playas cuya superficie disminuyó aún más a consecuencia de la construcción del puerto (a principios del siglo XX) y de las obras de defensa costera en el área central que protegieron unas playas a costa de otras.

A lo largo del tiempo es posible reconocer dos grandes períodos: una temprana desvalorización (fines del siglo XIX hasta la década de los '80) y una incipiente valorización que comienza en la década de los '8o.

En la primera etapa este sector fue sistemáticamente marginado de los procesos de valorización turística, esto es puesto en evidencia por las escasas y tardías obras de defensa costera y de accesibilidad a playas, la instalación sucesivamente de puntos de descarga de efluentes y la mínima dotación de equipamiento turístico.

Recién en la década de los ' 80 , cuando ya todos los tramos costeros de la ciudad estaban intensamente urbanizados y congestionados, el municipio torna la mirada hacia el tramo más cercano a la zona central llamando a licitación para la construcción del Complejo La Perla.

El resto de la costa ubicado hacia el norte recién será licitado -en forma discontinua y desigual- hacia fines de los '9o. En paralelo desde el Estado Municipal y Provincial se invierte en obras de defensa costera que restituyen arena a las deterioradas playas del norte.

En las últimas décadas, con la materialización de nuevas obras de tratamiento de aguas residuales y la construcción del efluente submarino, se abre una posibilidad de mejora en la calidad ambiental de las playas del norte.

Desde el municipio -incentivado por la provincia de Buenos Aires- se muestra un mayor interés por proveer servicios de mayor calidad a este sector. Cabe preguntarse ¿Qué nuevos actores sociales dominarán en este nuevo escenario? 


\section{Referencias bibliográficas}

Cicalese, G. (200I). Apertura democrática, gobierno local y políticas urbanas. Nueva apuesta a la construcción de Mar del Plata Balnearia en la década del «8: el caso del complejo balneario La Perla. En Revista FACES, 7(I2), Fac. Ciencias Ecómicas. UNMDP.

Gasperotti, L. (2018). Tratamiento de aguas residuales y turismo en las playas del Norte de Mar del Plata. Una mirada en dos temporadas estivales (20152016 y 20I6-20I7). Actas I Jornadas Nacionales de Geografía de la UNMDP, Mar del Plata.

Lagrange, A. (1993). Mar, Playas y Puerto, Mar del Plata: Editorial Fundación Bolsa de Comercio.

Morrell, P. A. (2002). Un aporte para la caracterización ambiental del litoral marítimo marplatense. En Actas Congreso Nacional de Geografia y 63 Semana de Geografía. GAEA, Buenos Aires.

Municipalidad de General Pueyrredón. Boletines Municipales. Toda la colección.

Pastoriza, E. (20II). La conquista de las vacaciones. Breve historia del turismo en la Argentina, Buenos Aires: Edhasa.

Rigonat, M. (2012). Urbanizaciones turísticas y concentración espacial del deterioro ambiental. El caso de la costa marplatense (Argentina) en Revista Geográfica Venezolana, nro. 53 (I) Enero-junio. Mérida-Venezuela. Universidad de los Andes.

Sanchez, J. (I99I). Espacio, economía y sociedad. Madrid. España: Editorial siglo XXI.

Sebreli, J. (1970). Mar del Plata, el ocio represivo. Buenos Aires: Editorial Leonardo Buschi.

Siragusa, A. (1984). Las condiciones naturales. Un marco natural de privilegio, en Roccatagliata, J. (coord.) Mar del Plata y su región. Buenos Aires: Editorial Sociedad Argentina de Estudios Geográficos.

Villar, M.d.C. (200o). Fronteras Terrestres de los Espacios Costeros. Reflexiones sobre su Variabilidad espacio-temporal en ciertos tramos del Litoral Marítimo Bonaerense. En 2das Jornadas Platenses de Geografía. Departamento de Geografía. Facultad de Humanidades y Ciencias de la Educación. La Plata. Universidad Nacional de La Plata. 